TITLE:

\title{
Asynchronous Co-eating Through Video Message Exchange: Support for Making Video Messages
}

$\operatorname{AUTHOR}(S)$ :

Obata, Kanako; Nakamura, Yuichi; Chen, LongFei; Augeri, John

\section{CITATION:}

Obata, Kanako ...[et al]. Asynchronous Co-eating Through Video Message Exchange: Support for Making Video Messages. Cross-Cultural Design. Applications in Health, Learning, Communication, and Creativity 2020: 338-348

ISSUE DATE:

2020

URL:

http://hdl.handle.net/2433/265004

\section{RIGHT:}

This is a post-peer-review, pre-copyedit version of an article published in Combinatorial Optimization and Applications. The final authenticated version is available online at: http://dx.doi.org/10.1007/978-3-030-49913-6_29; The full-text file will be made open to the public on 10 July 2021 in accordance with publisher's 'Terms and Conditions for SelfArchiving'.: This is not the published version. Please cite only the published version.この論文は出版社版でありません 。引用の際には出版社版をご確認ご利用ください 


\title{
Asynchronous Co-Eating through Video Message Exchange: Support for Making Video Messages
}

\author{
Kanako Obata ${ }^{1}$, Yuichi Nakamura ${ }^{10000-0001-5103-7194]}$, LongFei Chen ${ }^{1}$, and \\ John Augeri ${ }^{2}$ \\ 1 Academic Center for Computing and Media Studies, Kyoto University, \\ Sakyo, Kyoto, Japan \{obata, yuichi\}@media.kyoto-u.ac.jp \\ 2 Universit Numrique Paris Ile-de-France, 90 rue Tolbiac, 75634 Paris cedex 13, \\ Paris, France, john.augeri@unpidf.fr
}

\begin{abstract}
Co-Eating, i.e., eating meals in the company of other people, has been widely recognized as good for both physical and mental health. However, the chances of co-eating have drastically decreased for families living separately and for older people living alone. To cope with this problem, we propose a framework of "asynchronous co-eating" that enables virtual co-eating through video message exchanges, which does not necessarily require people to eat simultaneously. This framework is aimed to maintain frequent communication between family members and promote ordinary types of co-eating when possible. To make this process easy for both older people and their distant family members, we designed a video message exchange scheme with an omnidirectional camera and a topic recommendation mechanism. With preliminary experiments, we obtained results that suggest our framework has the potential to be beneficial.
\end{abstract}

Keywords: co-eating · communication support · health and mental care - gerontology

\section{Introduction}

It is widely acknowledged that co-eating, i.e., eating meals with other people, particularly those with whom we have close relationships, is good for both physical and mental health[1]. Co-eating provides good opportunities for communication among families, friends, and other members of the community for introducing, educating, and maintaining food and dietary cultures[2][3][4]. Furthermore, coeating allows for participants to be mutually aware of one another and potentially helps maintain good dietary habits and health by encouraging people to consume sufficient nutrition, but not too much.[5][6]. In recent years, however, chances of co-eating have been decreasing due to the increasing number of small families and people living alone; solitary eating is often seen among older people.

With this in mind, our research is aimed at providing virtual co-eating opportunities through the use of information and communication technology (ICT), 
and consequently helping to cope with the social problems of older people living alone and families living separately. Supporting communication while eating has much potential for alleviating some of these social problems, because eating and taking time to eat is essential for every person. During meals, people are often relaxed and have enough time to think of other people, especially family members.

As part of previous research on this topic, Tokunaga, et al. proposed a framework of co-eating through video conference[7]. They did experiments wherein two groups of family members living separately - on one side, older parents and on the other, their son or daughter and his/her partner and children-each ate their meals while watching and chatting with each other. The results of this experiment revealed that the older participants' quality of life was much improved, in particular their pleasure and interest in life due to their curiosity about discovering new things to serve as topics for conversation.

Although this type of simultaneous co-eating has many benefits, we often have difficulties for sharing time in this way. Family members living separately usually have different schedules, with, for example, older people tending to eat early in the evening and working people more likely to have quick meals later at night. As a result, it is often challenging for each group to adjust their time and way of eating. Surely, therefore, we need ideas on how to fill the gap between synchronous co-eating and eating alone.

To this end, we propose a framework of "asynchronous co-eating" that enables virtual co-eating through video message exchanges, which does not require people to eat simultaneously. This framework is not intended to replace synchronous co-eating, but to ease the difficulties of simultaneous eating, maintain communication, and promote ordinary types of co-eating when possible. Moreover, asynchronous co-eating also stimulates communication among family members who live together but have different schedules and do not regularly have meals together.

\section{Video Message Exchange for Co-Eating}

Our scheme of asynchronous co-eating is organized as follows. Suppose that $\mathrm{X}$ and $\mathrm{Y}$ are partners, each of them has a mobile device that can record video messages, upload or download them to or from cloud file servers, and replay downloaded video messages. Using these functions, $\mathrm{X}$ and $\mathrm{Y}$ record video messages as illustrated in Figure 1.

- X or Y records video messages with the device and uploads them to a cloud folder they share. Messages can be captured while executing everyday activities at home or anytime and anywhere.

- X or Y downloads messages from their counterpart and views them while they have their meal. Video responses also can be recorded while watching downloaded messages. 


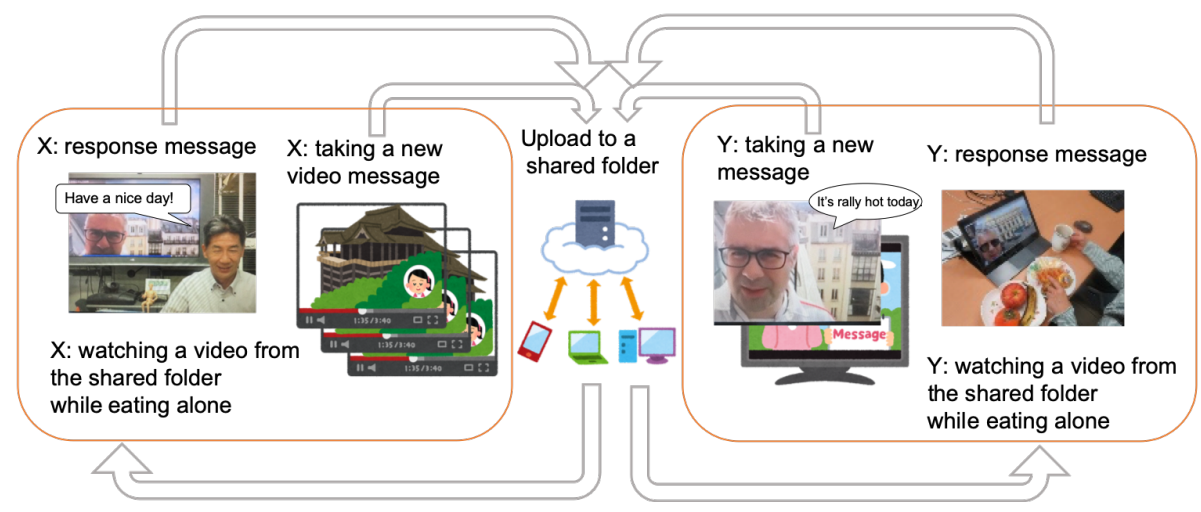

Fig. 1. Framework of asynchronous co-eating

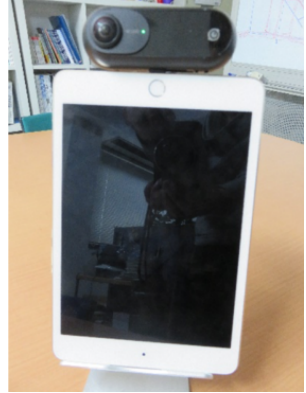

(a) recording device

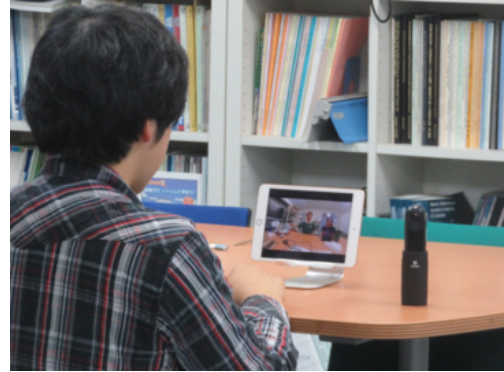

(b) recording watching situation

Fig. 2. Video recording device with omnidirectional camera attached

Thus, both $\mathrm{X}$ and $\mathrm{Y}$ can upload a new video message and reply to their partner's messages. Viewing video messages and possibly replying to them while eating is the crux of our framework for asynchronous co-eating. We assume that one of the most important users of this system would be older people living apart from their children or intimate individuals and the another type of users would be persons between 20 to 60 years old who have busy work lives. Therefore, we need to consider some problems caused by their living styles and differences.

\section{usability:}

- The device and user interface needs to be suitable for use by elderly people and while eating.

- The process should not require undue time and efforts for daily use.

\section{communication:}


- Motivation for making video messages is necessary because older people tend to have fewer events and changes in their lives compared to younger individuals. It is possible that their messages could potentially contain complaints about their lives or environments.

- Suppression of complaints and unpreserved expressions is necessary as these kinds of messages often cause worry or annoyance, which further discourages video message communication.

- To compensate for the lack of real-time interactions, responding is encouraged.

The problems of usability are primarily dealt with via the design of the device and its software.

\section{Design and Challenges}

One of the challenges of this research was assisting users, especially older people, to create good-quality video messages. To facilitate this, we designed the message acquisition scheme as follows:

- The communication device is equipped with an omnidirectional camera that takes images of not only what the operator or sender wishes to shoot but also of how the person behaves while recording the video (Figure 2). This is significant because we often feel that seeing the face of the family member is as important as it is to see what they are shooting.

- The length of video messages is deliberately kept short, e.g., 30 seconds or 1 minute, making both recording and watching easy.

- We also designed a topic selection assistance function that makes it easier for users to choose topics that are interesting and/or acceptable to their counterparts; this is enabled by aggregating the preferences of both sides and introducing random selections.

As mentioned above, because the face of the operating person, i.e., the message sender, is important to the message receiver, we need to detect the face and ensure that it is properly included in the final, edited video. For this purpose, we implemented a face tracking program that detects a face and tracks it with the Kalman filter based on a common face detection software[8].

Figure 3 illustrates examples of image composition from omnidirectional videos. (a) is a panoramic view that is most similar to a captured video, (b) is a horizontally merged view of the front and the back view, (c) is a frontal view with the face superimposed, for which the size and position can be changed, and (d) is a merged view wherein the front and back view are vertically aligned. For (b) and (d), an omnidirectional video is split into both front and back views based on the position of the camera's operator face, which is then merged horizontally or vertically. For (c), the area of the image around the detected face is superimposed in the front view. Because each of these views has advantages and disadvantages, we asked participants for subjective impressions for them. The results are shown in a later section. 


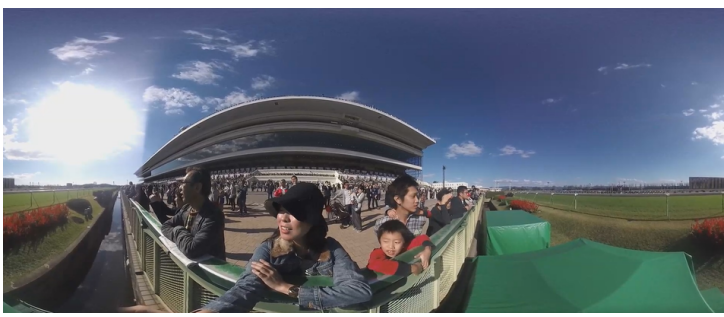

(a) panoramic view

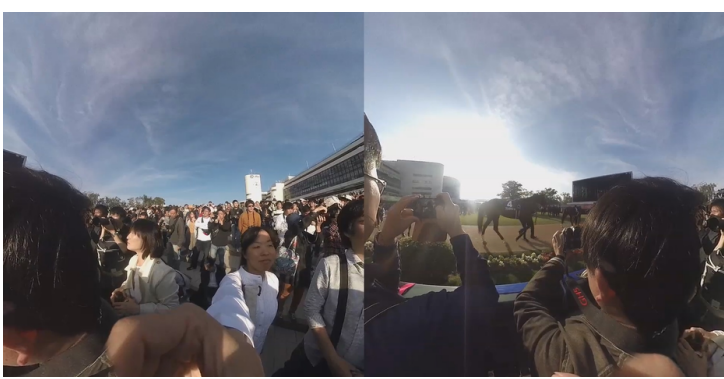

(b) split-and-merge view (horizontal)

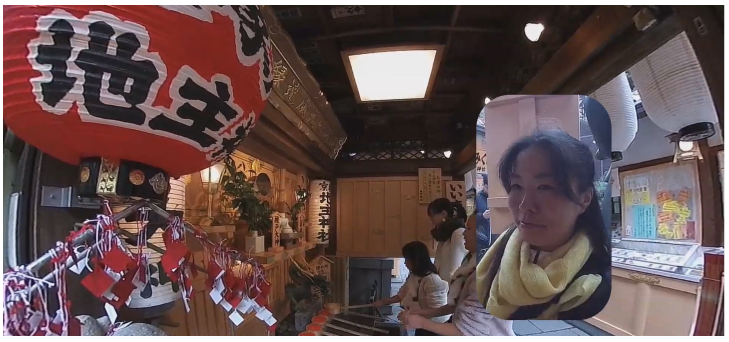

(c) superimpose view

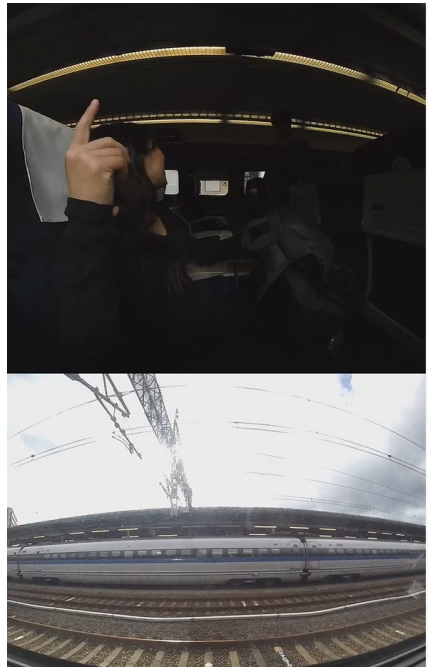

(d) split-and-merge view (vertical)

Fig. 3. Videos edited to show the face of the person recording it

\section{Support for Topic Selection}

Candidates for potential topics were arranged into the following groups:

(i) Topics on which the sender (the person recording) can easily make a message.

(ii) Topics that the receiver (the person viewing) is interested in.

(iii) Topics on current or seasonal events.

(iv) Requests for responses to previous messages.

Group (i) contains topics on hobbies, meals, family histories, etc., which encourage the sender to record the message. A small number of topics dealing with health problems and complaints on current problems, which may discourage communication, can be included. Group (ii) comprises topics that the receiver 
Table 1. Topic selected in the experiments

\begin{tabular}{c|r|r} 
& without roulette & with roulette \\
\hline health condition & $11(8 \%)$ & $6(5 \%)$ \\
weather & $7(5 \%)$ & $5(4 \%)$ \\
daily activity & $62(45 \%)$ & $25(20 \%)$ \\
family-related topic & $8(6 \%)$ & $2(1.6 \%)$ \\
events & $7(5 \%)$ & $4(3 \%)$ \\
current news & $10(7 \%)$ & $2(1.6 \%)$ \\
others & $32(23 \%)$ & - \\
chosen topic by the counterpart & 0 & $56(45 \%)$ \\
response to a previous message & 0 & $25(20 \%)$ \\
\hline
\end{tabular}

wants to see or hear from the sender. These topics are suggested by the receiver beforehand. It is difficult for both the sender and receiver to choose a potential topic every time in Group (iii), for which the system provides based on news and other public sources. Group (iv) accelerates dense communication on some topics and gives a feeling of interactive communication.

The sender may feel that he/she is forced to use the selected topic if an actual topic is simply specified. Our system intends to reduce this feeling of being forced by introducing randomness using a roulette. The sender uses the roulette to randomly select a topic from the above-mentioned topic groups. We expect that both the sender and receiver will be more comfortable without feeling the responsibility of choosing a topic.

\section{Preliminary Experiments}

\section{Topic Selection}

We asked five participants to record video messages on five topics each day for at most ten days. We asked the participants to record video messages based on their arbitrary choices in the first experiment. Chosen topics are listed in the left column of Table 1. Dominant topics were health conditions, weather, daily activity, current news, events, family-related, etc. Without specified topics, the participants mostly tried to find something new in everyday life, which was sometimes a considerable burden. In fact, this caused one participant to drop out without completing the task.

We asked the participants to record video messages with the topic suggested by the roulette in the second experiment. The number of topics recorded per day and the period for which they were recorded were the same as in the first experiment. Topic groups were selected based on the method mentioned in the previous section, taking into consideration the topics chosen in the first experiment. Chosen topics are listed in the right column of Table 1. Distribution of the topics was roughly controlled by the candidates and randomness. As a result, "response to a previous message" is chosen at a rate of $20 \%$. 
Table 2. Comparison of with and without roulette

\begin{tabular}{c|r|r} 
& without roulette & with roulette \\
\hline New findings & $17(12.4 \%)$ & $34(27 \%)$ \\
Complaints & $14(10 \%)$ & $6(4.8 \%)$ mostly light (not serious) \\
\hline
\end{tabular}

Table 3. Evaluation of video messages (how interesting it is)

\begin{tabular}{l|r|r} 
score & without roulette & with roulette \\
\hline 1 (not interesting) & 0 & 0 \\
2 & $11(8 \%)$ & $1(1 \%)$ \\
3 (neutral) & $95(69 \%)$ & $55(44 \%)$ \\
4 & $29(21 \%)$ & $55(44 \%)$ \\
5 (quite interesting) & $2(1.5 \%)$ & $14(11 \%)$ \\
\hline
\end{tabular}

Concerning the content of the video messages, we asked the participants what new thing did they learn about their counterparts. This evaluation did not concern the topic content, but rather the characteristics, ideas, daily life, or anything else about the message senders. Table 2 demonstrates the comparison of the first and second experiments. The proposed method apparently has benefits for eliciting new information that family members did not have beforehand. It also suppresses serious complaints that may worry the receivers. Table 2 shows that the number and quality of complaints decreases when using this method.

Another feature of this proposed method is that it occasionally leads to message content that the receiver does not expect. For example, one participant asked about "animals," considering this to mean a pet, but he received a message concerning a mosquito and its bites. This type of unexpected message exchange contributes to the sharing of new information among families.

As another aspect of content evaluation, we asked the participants to evaluate video messages based on how interesting they were. Table 3 compares the results of the first and second experiments; scores are marked from 1 (nothing interesting) to 5 (quite interesting). It can be seen that the proposed method made messages more acceptable to the receivers.

As for the difficulty in making video messages, we asked the participants to score it between 1 (easy) and 5 (difficult). The result suggests that the proposed method makes the task easier.

Table 4. Difficulty in making video messages (Figures in the two columns on the right indicate the number of participants)

\begin{tabular}{l|r|r} 
score & without roulette & with roulette \\
\hline 5 (difficult) & 1 (dropped out) & 0 \\
4 & 2 & 1 \\
3 (neutral) & 2 & 1 \\
2 & 0 & 0 \\
1 (easy) & 0 & 2 \\
\hline
\end{tabular}


Table 5. Comments on topic selection

- It is difficult to find fresh topics every day by myself.

- Topic selection makes me notice that my daily life lacks variety and tends to be trivial.

- It was easy for me to make video messages on the specified topics chosen by the roulette.

- Choosing topics by myself is easier.

- Making video messages is like writing in a diary.

- The topics need to be more varied.

- I wish to record messages based on some topics that were not chosen.

Table 6. Impressions and comments on the scheme

- It is easier to record a video message than talk on the telephone or face-to-face.

- It is easier to view a video message than to talk on the telephone or face-to-face.

- I noticed everyday things a bit more carefully because I needed to make a message for someone.

- Recording video messages is fun, as if I am chatting with a friend.

- It is difficult to talk to a camera.

- I hesitate to record video messages in a crowded space.

- To watch daily messages is more fun than I previously expected. Even ordinary content can be interesting and fun.

- Seeing the face of the sender is often more important than what they are saying and makes for good content in itself.

Table 5 indicates the comments given by the participants. It suggests that the proposed method makes topic selection easier. However, one participant felt it would be easier to choose a topic on his own. It may be necessary to increase the rate of a roulette result of "free topic," to allow users to arbitrarily select topics. We also need to consider topic variations, and how the users expect them. Table 6 also shows the impressions and comments on the entire scheme of asynchronous co-eating. The comments suggest that our scheme has considerable advantages.

\section{Video Composition}

To clarify the characteristics of possible video compositions, we asked the participants to state their impressions after viewing the edited message videos. Table7 presents viewers' common impressions. For the comparison, baseline video compositions were also considered, i.e., ordinary composition with the camera pointing at a target and taking a "selfie."

The impressions were practically what we expected. Ordinary video compositions without the message sender in the frame or "selfie" lack information and do not stimulate responses. Among the composition from omnidirectional video, 
Table 7. Impressions and comments on the image compositions. Actual samples are presented in Figure 3.

Panoramic view, e.g., Figure3 (a)

- Difficult to view and understand the scene.

- It has unpleasant distortion.

- Looks like a mirror.

- This may be unsuitable or difficult for older people to view as it requires skills.

Split-and-merge view, e.g., Figure 3 (b), (d)

- Easy to understand where and how the person recording the message is.

- It is mostly good if the message sender is not moving fast.

- It is sometimes confusing if both the front and back views are similar, e.g., Figure $4(\mathrm{a})$.

- It stimulates replying because it is easily understandable.

Superimposed view, e.g., Figure 3 (c)

- It feels like a television screen.

- A larger image of the message sender is preferred. Faces in samples are too small, e.g., Figure 3 (c)

- Want to see the body and clothes.

- It is mostly good if the message sender is not moving fast.

- Difficult to see what the sender is looking at if he/she frequently turns the head, e.g., Figure 4(a).

- The superimposed face stimulates response.

Baseline (ordinary shot and selfie), e.g., Figure 4 (a) and (b)
- Easy to watch, but boring (both)
- Want to see the sender's face/body (the case of ordinary view).
- Want to see the surrounding environment (both)

the split-and-merge view and the superimposed view were better received than the panoramic view. The panoramic view could contain a lot of information of the sender's surroundings; however, images captured this way were extremely distorted and the sender's face was too small. This made viewing videos captured in this view difficult and weakened the feeling of interaction on the part of the receiver. Therefore, we consider both the split-and-merge and superimposed views to be the primary candidates of video composition in our model.

That said, it may also be true that what constitutes suitable composition and editing can differ depending on the situations and environments wherein videos are captured, and, of course, according to personal preferences. We need to further investigate automatic video composition. 


\section{Summary}

We introduced a novel framework of asynchronous co-eating to stimulate communication with loved ones for older people and separated family members. First, we discussed the problems preventing eating together and the idea of video message exchange targeting virtual co-eating. Second, we present the scheme and design of our asynchronous co-eating system, concentrating on device design and the support for topic selection. Our preliminary experiments indicated good potential of our scheme, although we need systematic evaluation with a higher number of participants. Prototyping of the total framework must be undertaken in the future, in which enables a more realistic evaluation of the system with a variation in the older people and families participating.

\section{References}

1. "Healthy Eating Habits: Benefits Of Eating Together With Family And Friends", "https://www.flavoursholidays.co.uk/blog/healthy-eating-habits-why-eating-withfriends-and-family-keeps-you-young/", last accessed, 27, Jan., 2020

2. James Bossard, "Family Table Talk - An Area for Socialogical Study", American Sociological Review, Vol.8, No.3, pp.295-301, 1943

3. Sheila Gahagan, "Development of eating behavior: biology and context", Journal of Developmental and Behavioral Pediatrics, Vol.33, No.3, pp.261-71, 2012

4. Robin Dunbar, "Breaking Bread: the Functions of Social Eating", Adaptive Human Behavior and Physiology, Vol.3, pp.198-211, 2017

5. Ryuzaburo Nakata, Nobuyuki Kawai, "The 'social" facilitation of eating without the presence of others: Self-reflection on eating makes food taste better and people eat More", Physiology \& Behavior, Vol. 179, pp. 23-29, 2017

6. Hiroko Tokunaga, Naoki Mukawa, Atsushi Kimura, "Structural differences of eating behavior between solitary eating and co-eating", Journal for the Integrated Study of Dietary Habits, Vol.27, No.3, pp. 167-174, 2017

7. Haruka Konno, Hiroko Tokunaga, Naoki Mukawa, "Influence of Video-mediated Co-eating Communication between Elderly Parents and Their Independent Child on Meal Satisfaction and Quality of Life" (In Japanese), IEICE SIG-HCS, Vol.117, No.29, pp.265-270, 2017

8. Adrian Bulat and Georgios Tzimiropoulos, "How far are we from solving the 2D \& 3D Face Alignment problem? (and a dataset of 230, 000 3D facial landmarks) ", International Conference on Computer Vision ("http://arxiv.org/abs/1703.07332"), 2017 


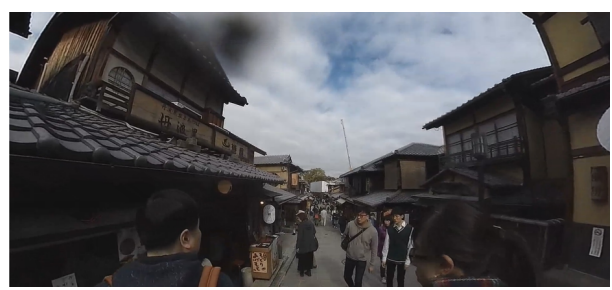

(a) ordinary shot

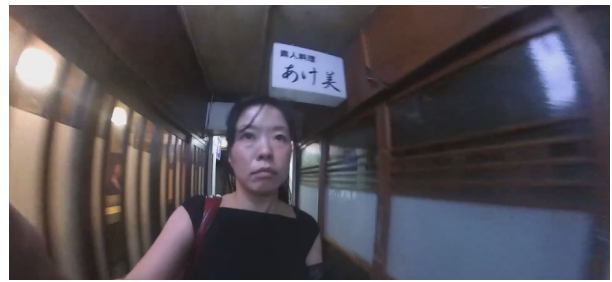

(b) selfie

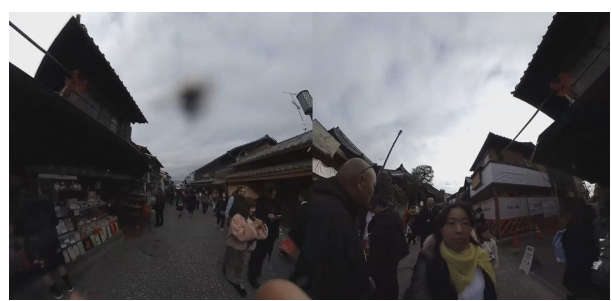

(c) forward and backward views are similar

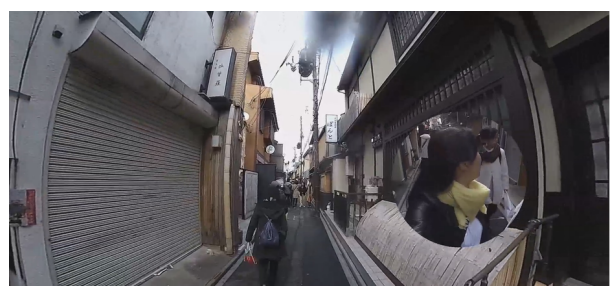

(d) difficult to find gazing target

Fig. 4. Supplemental samples for the impressions in Table 7 